\title{
Capacidade de Brotação de Teca sob Regime de Talhadia Composta
}

\author{
Kamila Lemos Costa Barros ${ }^{1}$, Renato Luiz Grisi Macedo ${ }^{1}$, Nelson Venturin ${ }^{1}$ \\ ${ }^{1}$ Departamento de Ciências Florestais, Universidade Federal de Lavras - UFLA, Lavras/MG, Brasil
}

\begin{abstract}
RESUMO
O objetivo do trabalho foi avaliar a capacidade de brotação de cepas de teca sob regime de talhadia composta. O experimento foi implantado nos espaçamentos 3,0 $\times 2,0 \mathrm{~m}$ e 3,0 $\times 3,0 \mathrm{~m}$. Aos 8 anos de idade, o experimento foi desbastado sistematicamente, em $50 \%$. Após o desbaste, foi avaliado o potencial de brotação das cepas das árvores desbastadas. O delineamento experimental utilizado foi o de blocos ao acaso, com 2 tratamentos e 5 repetições. Os tratamentos foram T1 = brotação conduzida sob regime de talhadia composta no espaçamento 3,0 × 4,0 m e T2 = brotação conduzida sob regime de talhadia composta no espaçamento $3,0 \times 6,0 \mathrm{~m}$. A teca apresentou capacidade de brotação de cepas sob regime de talhadia composta até o período avaliado de 12 anos de idade, para ambos tratamentos.
\end{abstract}

Palavras-chave: espaçamentos, sombreamento, brotações.

\section{Teak Stump Sprouting Capacity Under Compound Coppice Regime Process}

\begin{abstract}
This work aimed to evaluate the teak stump sprouting capacity under compound coppice process. The experiment was implemented at spacing of $3.0 \times 2.0 \mathrm{~m}$ and $3.0 \times 3.0 \mathrm{~m}$. At 8 -years old, the experiment was systematically thinned in 50\%. After thinning, with new spacing of $3.0 \times 4.0 \mathrm{~m}$ and $3.0 \times 6.0 \mathrm{~m}$, the stump sprouting capacity of the thinned trees was evaluated. The experimental design used was randomized blocks, whit two treatments and 5 repetitions. The treatments were $\mathrm{T} 1=$ conduced sprouting under compound coppice process at the spacing $3.0 \times 4.0 \mathrm{~m}$ and $\mathrm{T} 2=$ conduced sprouting under compound coppice at the spacing $3.0 \times 6.0 \mathrm{~m}$. Teak presented sprouting capacity under compound coppice until 12-years old for both treatments.
\end{abstract}

Keywords: spacings, shading, stumps. 


\section{INTRODUÇÃO}

A teca é uma espécie oriunda do sudeste asiático bem adaptada às condições edafoclimáticas do Brasil, principalmente na Região Centro-Oeste do país. Além das condições climáticas favoráveis, o solo de melhor fertilidade e os tratos silviculturais mais adequados e intensos contribuíram para reduzir o ciclo de produção da espécie de 80 anos, na região de origem da teca, para apenas 25 anos, na região de Cáceres, MT (Costa, 2011).

A madeira da teca é utilizada para diversos fins, como laminação e compensados, lenha e carvão vegetal, sendo que os dois últimos são mais comuns para as áreas de ocorrência natural. A madeira da teca é estável e a sua principal utilização é na construção naval, pois apresenta características de alta estabilidade quando em contato com a água do mar (Caldeira \& Oliveira, 2008; Costa, 2011).

Para tanto, o manejo dessa espécie e o conhecimento de suas características silviculturais são ferramentas de suma importância para o sucesso de um povoamento implantado de teca, bem como para obtenção de lucros e retornos financeiros satisfatórios.

O método de talhadia simples é o que se origina vegetativamente a partir de brotos das cepas ou tocos deixados pelas árvores cortadas. O regime de talhadia composta é uma variação do sistema de talhadia simples no qual populações sob regeneração a partir de brotações de cepas são formadas sob o dossel superior, composto por árvores advindas da regeneração por meio de sementes ou mudas.

Em síntese, o sistema é uma mistura de árvores formadas a partir da brotação das cepas crescendo sob um dossel constituído por árvores formadas pela reprodução de alto fuste, resultando em produtos de várias dimensões.

Os tratos silviculturais nesse tipo de regime devem se estender por todo o período de desenvolvimento de ambos os estratos, compreendendo as seguintes atividades: limpeza, desbaste e poda.

O regime de talhadia composta fornece material de diferentes dimensões, suprindo a demanda local de materiais de pequeno, médio e grande porte (lenha, poste, madeira para serraria etc.); providencia uma melhor cobertura do solo e, consequentemente, minimiza os danos por erosão, além de promover grande diversidade de estratos.

Todavia, as desvantagens da adoção desse regime são: dificuldade de ser aplicado correntemente; o crescimento do sub-bosque abaixo do dossel superior é em geral menos vigoroso do que na talhadia simples; a colheita do produto final requer mais trabalho do que sob o sistema de talhadia simples.

A teca apresenta uma boa capacidade de rebrota após desbastes e, de acordo com Gonzaléz (2004), em plantios desbastados, as brotações mostram um crescimento acelerado que concorre com o das árvores que ainda estão em pé. De acordo com o mesmo autor, a teca tem a capacidade de emitir brotações vigorosas nas cepas.

O maior crescimento inicial das brotações se deve à utilização de reservas orgânicas e inorgânicas na cepa ou nas raízes, e, posteriormente, quando passam a depender mais diretamente do solo, da existência de um sistema radicular desenvolvido que favorece a absorção de água e nutrientes, aumentando a taxa de crescimento da parte aérea das brotações (Oliveira et al., 2008).

Em razão de se ter elevada relação raiz/parte aérea, os assimilados são alocados preferencialmente para a formação da parte aérea, aumentando, assim, as diferenças na taxa de crescimento em relação às plantas da primeira rotação. A taxa de crescimento das brotações, na sua fase inicial de crescimento, é elevada em comparação com o crescimento de plantas provenientes de mudas (Reis \& Reis, 1997).

A hipótese do presente trabalho é a de que a teca apresenta capacidade de crescimento das brotações de cepas sob regime de talhadia composta. Inserido nesse contexto, o objetivo do presente trabalho foi avaliar a capacidade de brotação de cepas de Tectona grandis $\mathrm{L}$. f. (teca), sob regime de talhadia composta implantada no município de Paracatu, região noroeste de Minas Gerais.

\section{MATERIAL E MÉTODOS}

\subsection{Caracterização geral da área}

O experimento foi instalado na empresa Votorantim Siderurgia, na região noroeste do Estado de Minas Gerais, no município de Paracatu, localizado a $17^{\circ} 36^{\prime}$ de 
latitude sul e $46^{\circ} 42^{\prime}$ de longitude oeste, em altitude aproximada de $550 \mathrm{~m}$.

Segundo Antunes (1986) e Brasil (1992), o clima da região é o tropical úmido de savana, com inverno seco e verão chuvoso, do tipo Aw, conforme a classificação de Köppen. A temperatura média anual é de $22,6^{\circ} \mathrm{C}$, tendo uma média mensal de $18{ }^{\circ} \mathrm{C}$ na estação mais fria e de $29,1^{\circ} \mathrm{C}$ na mais quente. A precipitação média anual é de $1.450 \mathrm{~mm}$, apresentando médias mensais inferiores a $60 \mathrm{~mm}$ nos meses mais secos.

Os solos ocorrentes na região são classificados como Latossolo Vermelho-Escuro (na margem dos rios Grande e Parnaíba), Latossolos Vermelho-Amarelo, Areias Quartzosas e Solos Aluviais (Brasil, 1992). O solo predominante na área experimental é do tipo Latossolo Vermelho Amarelo distrófico (Macedo et al., 2005).

\subsection{Caracterização do experimento}

O experimento foi implantado inicialmente no mês de dezembro de 1997, nos espaçamentos 3,0 × 2,0 m (1.666 árvores.ha ${ }^{-1}$ ) e 3,0 × 3,0 m (1.111 árvores.ha $\left.{ }^{-1}\right)$. Aos 8 anos de idade, o experimento foi desbastado sistematicamente, em 50\%, no mês de julho, época de estiagem na região. Após o desbaste, nos novos espaçamentos, de 3,0 × 4,0 m e 3,0 ×6,0 m, foi avaliado o potencial de brotação das cepas das árvores desbastadas.

Na Tabela 1 estão registrados os valores médios de $\operatorname{DAP}(\mathrm{cm})$, altura total $(\mathrm{m})$, área seccional $\left(\mathrm{m}^{2}\right)$, área basal $\left(\mathrm{m}^{2} \cdot\right.$ ha $\left.{ }^{-1}\right)$, volume individual $\left(\mathrm{m}^{3}\right)$, calculado utilizando-se do fator de forma proposto por Macedo et al. (2005) e volume total com casca $\left(\mathrm{m}^{3} \cdot \mathrm{ha}^{-1}\right)$, para os espaçamentos iniciais de plantio $(3,0 \times 2,0 \mathrm{~m} \mathrm{e} \mathrm{3,0 \times 3,0} \mathrm{m)}$.

Aos 9 anos de idade (1 ano após o desbaste), para as cepas das árvores removidas sob regime de talhadia composta foi conduzido o broto mais vigoroso e de maior porte, eliminando-se os brotos excedentes.
Esses foram eliminados com o auxílio de um serrote, sendo cortados bem rentes à cepa.

O delineamento experimental utilizado foi o de blocos ao acaso, composto por dois tratamentos: $\mathrm{T} 1$ = Tratamento 1 , brotação conduzida sob talhadia composta no espaçamento $3,0 \times 4,0 \mathrm{~m}$, resultante do desbaste de 50\% no espaçamento 3,0 × 2,0 m e, $\mathrm{T} 2=$ Tratamento 2 , brotação conduzida sob talhadia composta no espaçamento $3,0 \times 6,0 \mathrm{~m}$, resultante do desbaste de $50 \%$ no espaçamento $3,0 \times 3,0 \mathrm{~m}$, com 5 repetições, avaliadas em quatro diferentes idades, aos 9, 10,11 e 12 anos.

Foram utilizadas unidades amostrais, com parcelas permanentes de área útil de 96,0 $\mathrm{m}^{2}$ (para T1) e 144,0 $\mathrm{m}^{2}$ (para T2), compostas por 8 plantas úteis/repetição/tratamento.

\subsection{Informações coletadas e análise do experimento}

Aos 9 anos iniciaram-se as medições das variáveis dendrométricas CAP (circunferência a altura do peito) e altura total do broto conduzido e a contagem dos brotos presentes nas cepas das árvores removidas.

As CAP foram obtidas com precisão de décimos de centímetro de todos os brotos conduzidos das unidades experimentais. Essa medida foi tomada com fita métrica sobre a posição da CAP (1,30 m do solo) e foi transformada em diâmetro a altura do peito (DAP), dividindo-se a CAP pela constante $\pi$. As alturas de todos os brotos conduzidos foram medidas inicialmente com réguas, com precisão de décimos de metro, e, posteriormente, usando-se hipsômetro Blume-Leiss.

Em cada uma das avaliações foram contados os brotos que cresciam nas cepas de teca advindos da regeneração sob talhadia composta. Após a contagem, os brotos presentes nas cepas foram cortados e lançados

Tabela 1. Valores médios de DAP, altura (H), área seccional (gm), área basal (G), volume individual (vm) e volume total com casca (V), aos 96 meses de idade de teca, para os espaçamentos inicias de plantio - Fazenda Riacho, Paracatu, MG.

Table 1. Average values of DBH, height $(\mathrm{H})$, cross sectional area (gm), base area $(\mathrm{G})$, individual volume (vm) and total volume with husk (V), in 96-months old teak for the initial planting spacings - Fazenda Riacho, Paracatu-MG.

\begin{tabular}{ccccccc}
$\begin{array}{c}\text { Espaçamentos } \\
\text { iniciais }\end{array}$ & $\begin{array}{c}\text { DAP } \\
(\mathbf{c m})\end{array}$ & $\begin{array}{c}\mathbf{H} \\
\mathbf{( m )}\end{array}$ & $\begin{array}{c}\mathbf{g m} \\
\left(\mathbf{m}^{2}\right)\end{array}$ & $\begin{array}{c}\mathbf{G} \\
\left(\mathbf{m}^{\mathbf{2}} \cdot \mathbf{h a}\right.\end{array}$ & $\begin{array}{c}\mathbf{v m} \\
\left(\mathbf{m}^{\mathbf{3}}\right)\end{array}$ & $\begin{array}{c}\mathbf{V} \\
\left(\mathbf{m}^{3} \cdot \mathbf{h a}\right.\end{array}$ \\
\hline $3,0 \times 2,0 \mathrm{~m}$ & 9,09 & 9,20 & 0,0070 & 11,7215 & 0,0159 & 26,4613 \\
$3,0 \times 3,0 \mathrm{~m}$ & 9,16 & 9,75 & 0,0072 & 8,0057 & 0,0263 & 29,2608 \\
\hline
\end{tabular}


ao solo para futura incorporação, com exceção do broto conduzido, selecionado inicialmente e demarcado por um fitilho colorido.

Para o cálculo do volume do broto conduzido foi utilizado o fator de forma com valor de 0,43 , proposto por Macedo et al. (2005).

O experimento foi medido e analisado aos 9, 10, 11 e 12 anos de idade, registrando-se informações sobre a dinâmica de crescimento e a produção da floresta para cada um dos tratamentos testados. As médias dos tratamentos foram comparadas por meio de regressão e Teste de Identidade de Modelos Lineares, a 5\% de probabilidade.

\section{RESULTADOS E DISCUSSÕES}

Por meio da análise do teste de identidade de modelos foi verificado que, estatisticamente, houve relação significativa entre as idades avaliadas e a emissão de brotos por cepa de teca sob regime de talhadia composta (Tabela 2).

Com vistas ao tratamento 1 (T1), foi observado que a dinâmica de crescimento das brotações da cepa da teca apresentaram uma tendência decrescente com o passar da idade, conforme apresentado na Figura 1.

Provavelmente, um dos fatores que contribuiu para essa tendência linear decrescente pode ser o início da competição intraespecífica entre o broto conduzido e as novas emissões de brotação. Como a competição por luz, água e nutrientes pode ter aumentado com o decorrer da idade, visto que o broto conduzido demandaria maior quantidade de recursos, a emissão de brotos tenderia a reduzir.

Macedo et al. (2005) verificaram, aos 36 meses de idade, que um plantio de teca na mesma região de estudo do presente trabalho apresentou resultados favoráveis ao crescimento da teca no espaçamento $3,0 \times 2,0 \mathrm{~m}$, mesmo demonstrando competição intraespecífica por luz, decorrente da maior densidade de plantas verificada nas linhas de plantio dispostas no sentido leste-oeste.

Em contrapartida, para o tratamento 2 (T2), a dinâmica de crescimento das brotações apresentou-se uniforme com o decorrer da idade, o que pode ser justificado pela época de realização do desbaste.

Assmann (1961) apud Schneider et al. (1998), analisando a reação dos povoamentos florestais a desbastes, observou que as árvores remanescentes aumentavam rapidamente o incremento, devido ao melhor aproveitamento dos fatores ambientais. Observou ainda que esse efeito, que denominou de Efeito de Aceleração do Crescimento, é dependente da época de aplicação do desbaste e do seu peso.

Conforme afirmações de vários autores (Chaves \& Fonseca, 1991; Salas \& González, 2003; Kanninen et al., 2004; Perez \& Kanninen, 2005; Bebarta, 2006; Cordero \& Kanninen, 2003), a realização de desbastes em teca para favorecimento do seu crescimento deve ser precoce, em povoamentos jovens, em média com 5 anos de idade, o que não ocorreu no presente caso, no qual o povoamento foi desbastado aos 8 anos de idade.

Em estudo realizado por Jerez et al. (2003), eles afirmam que regimes de espaçamentos mais adensados com desbastes frequentes parecem ser mais produtivos no que diz respeito ao rendimento total, em comparação aos regimes de espaçamento mais amplo e poucos desbastes.

Em média, aos 9 anos de idade, para T1, foram emitidos 8 brotos por cepa de teca e, para T2, 6 brotos (espaçamento mais amplo), o que contradiz vários autores (Salas \& González, 2003; Drescher, 2004;

Tabela 2. Análise de variância para a variável número de brotos, analisada aos 9, 10, 11 e 12 anos de idade de brotações de teca sob talhadia composta - Fazenda Riacho, Paracatu, MG.

Table 2. Variance analysis for the variable number of sprouts analyzed at 9, 10,11 and 12-years old sprouts under compound coppice - Fazenda Riacho, Paracatu-MG.

\begin{tabular}{lcrrc}
\hline \multicolumn{1}{c}{ Fonte de variação } & GL & SQ & QM & F \\
Modelo completo & 4 & 17,7337 & & $1,8860^{*}$ \\
Modelo reduzido & 2 & 7,8059 & 4,9639 & \\
Diferença & 2 & 9,9278 & 2,6319 \\
Resíduo & 16 & 42,1108 & \\
Total & 40 & 59,8445 & \\
\hline
\end{tabular}

* = valor de F calculado significativo a 5\%. GL = grau de liberdade; $\mathrm{QQ}=$ soma de quadrados; $\mathrm{QM}=$ quadrados médios. 
Gonzaléz, 2004; Passos et al., 2006), que afirmam que a teca é uma espécie heliófila e que o sombreamento interfere diretamente no seu crescimento.

Odecréscimo do número de brotos, independentemente do espaçamento utilizado, demonstra que os efeitos da competição intraespecífica pelos fatores de produção limitantes começam a se evidenciar. Reis \& Reis (1997), num estudo realizado com eucalipto, afirmaram que o rápido crescimento inicial de brotações de cepas pode resultar em obtenção de produção máxima mais cedo do que nos casos de primeira rotação.

A Figura 2 apresenta a dinâmica de crescimento do DAP dos brotos conduzidos por cepa de teca sob talhadia composta aos 9, 10, 11 e 12 anos de idade para os dois tratamentos. Observa-se que os tratamentos apresentaram uma tendência linear crescente nas curvas de crescimento em DAP com o decorrer da idade.
Para T2 ocorre uma maior inclinação da reta para a variável DAP que para T1, provavelmente, pela maior disponibilidade de luz, que favoreceu um melhor desempenho no crescimento em diâmetro dos brotos conduzidos. Há de se ressaltar, ainda, que a espécie possui um sistema radicular desenvolvido, que favorece a absorção de água e nutrientes, aumentando a taxa de crescimento da parte aérea (Oliveira et al., 2008).

Nas Figuras 3 e 4 pode ser observada a dinâmica de crescimento em altura e volume individual, respectivamente, dos brotos conduzidos por cepa de teca sob talhadia composta aos 9, 10, 11 e 12 anos de idade para os dois tratamentos. Observa-se que os tratamentos apresentaram uma tendência linear crescente nas curvas de crescimento, assim como para a variável DAP, com o decorrer da idade, o que corrobora a dinâmica natural ascendente de crescimento da maior parte das espécies florestais com o passar dos anos.
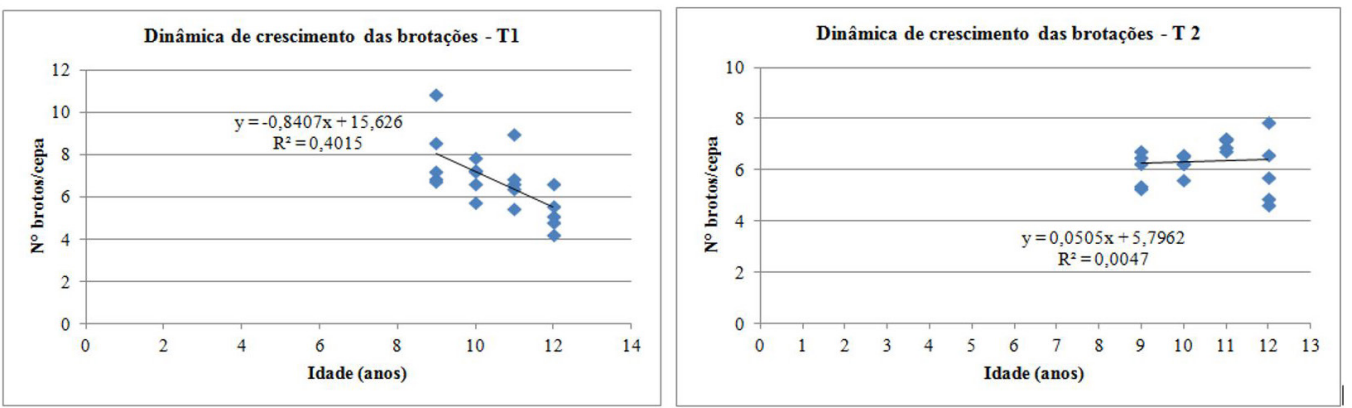

Figura 1. Dinâmica de crescimento - número de brotos por cepa de teca sob talhadia composta aos $8,9,10$ e 11 anos de idade para os tratamentos 1 e 2 - Fazenda Riacho, Paracatu, MG.

Figure 1. Growth dynamic of teca number of sprouts sprouts by stump under compound coppice at 8,9,10 and 11-years old for treatments 1 and 2 - Fazenda Riacho, Paracatu-MG.
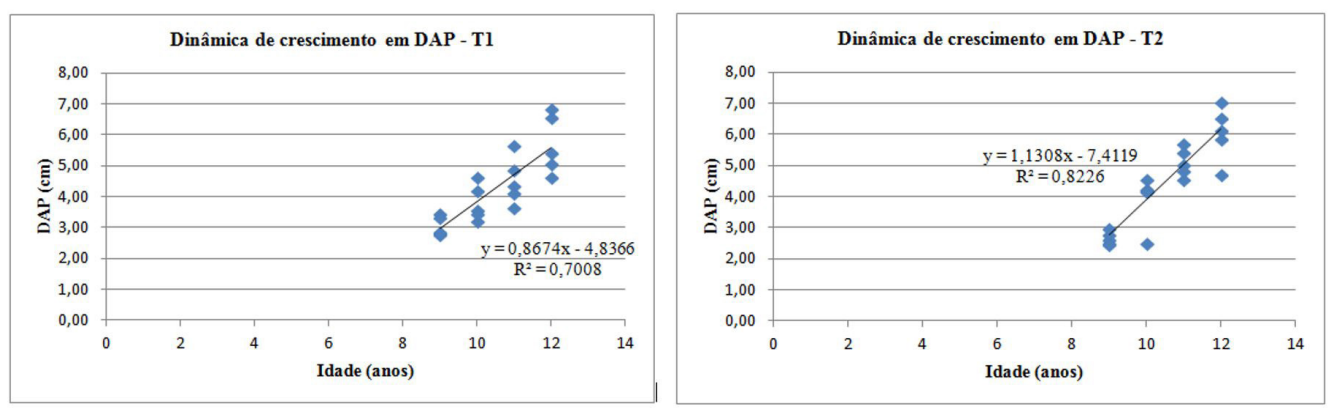

Figura 2. Dinâmica de crescimento do DAP dos brotos conduzidos por cepa de teca sob talhadia composta aos 9, 10, 11 e 12 anos de idade para os dois tratamentos - Fazenda Riacho, Paracatu, MG.

Figure 2. Growth dynamic of $\mathrm{DBH}$ of teca sprouts conducted by stump under compound coppice at 9, 10, 11 and 12-years old for both treatments - Fazenda Riacho, Paracatu-MG. 

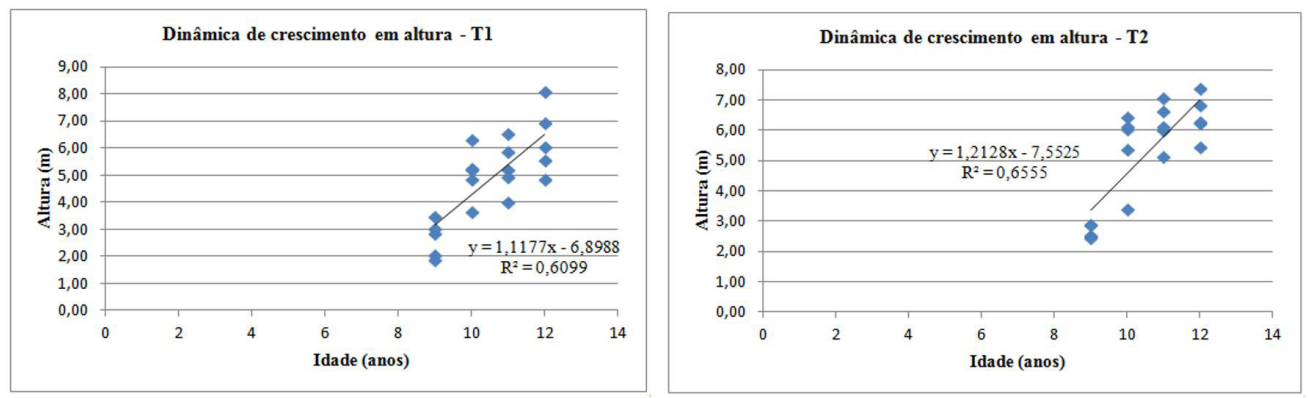

Figura 3. Dinâmica de crescimento em altura dos brotos conduzidos por cepa de teca sob talhadia composta aos 9, 10, 11 e 12 anos de idade para os dois tratamentos - Fazenda Riacho, Paracatu, MG.

Figure 3. Growth dynamic of plant height of teca sprouts conducted by stump under compound coppice at 9, 10, 11 and 12-years old for both treatments - Fazenda Riacho, Paracatu-MG.
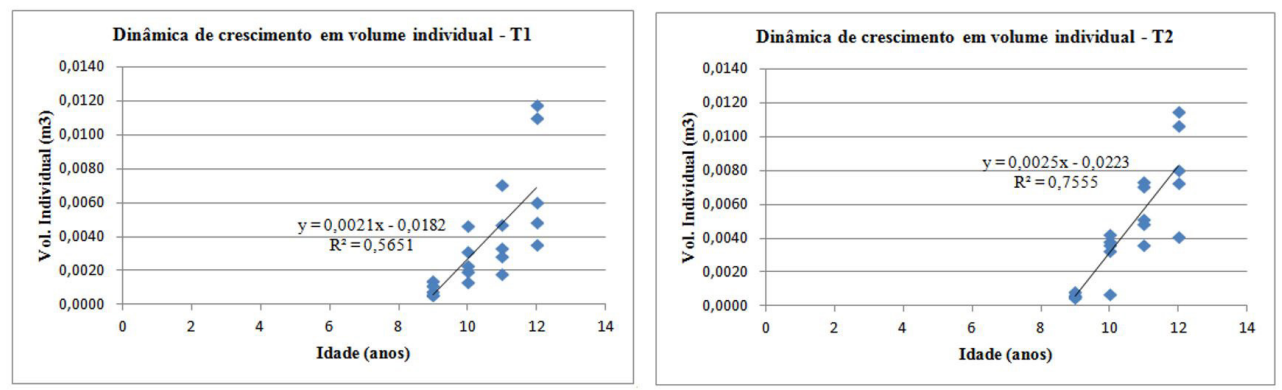

Figura 4. Dinâmica de crescimento em volume individual dos brotos conduzidos por cepa de teca sob talhadia composta aos 9, 10, 11 e 12 anos de idade para os dois tratamentos - Fazenda Riacho, Paracatu, MG.

Figure 4. Growth dynamic of volume per plant of teca sprouts conducted by stump under compound coppice at 9, 10, 11 and 12-years old for both treatments - Fazenda Riacho, Paracatu-MG.

\section{CONCLUSÕES}

A avaliação do número de brotos gerados ao longo dos anos nas cepas de teca, para o presente estudo, é um indicativo da capacidade de rebrota da espécie sob sombreamento, mesmo sendo vista como um complicador no processo de condução, elevando o gasto com desbrota, bem como agravante da desuniformidade do povoamento conduzido.

No espaçamento mais adensado, a emissão de brotações foi decrescente com o passar da idade, o que pode ser atribuído ao início da competição intraespecífica entre as brotações e o broto conduzido. Todavia, no espaçamento mais amplo, a emissão de brotações apresentou-se uniforme, podendo estar diretamente relacionada à época de realização de desbaste.

\section{AGRADECIMENTOS}

Os autores agradecem à Votorantim Metais e à Universidade Federal de Lavras.

\section{STATUS DA SUBMISSÃO}

Recebido: 25 fev., 2015

Aceito: 26 jun., 2016

\section{AUTOR(ES) PARA CORRESPONDÊNCIA}

\section{Kamila Lemos Costa Barros}

Instituto de Florestas, Universidade Federal Rural do Rio de Janeiro - UFRRJ, BR-465, Km 7, CEP 23897-000, Seropédica, RJ, Brasil e-mail: kafloresta7@gmail.com 


\section{REFERENNCIAS}

Antunes FZ. Caracterização climática do Estado de Minas Gerais: climatologia agrícola. Informe Agropecuário 1986; 12(138): 9-13.

Bebarta KC. Teak: ecology, silviculture, management, and profitability. Dehra Dun: International Book; 2006. 380 p.

Brasil. Ministério da Agricultura e Reforma Agrária. Departamento Nacional de Meteorologia. Normais climatológicas (1961-1990). Brasília: MARA; 1992.84 p.

Caldeira SF, Oliveira DLC. Desbaste seletivo em povoamentos de Tectona grandis com diferentes idades. Acta Amazonica 2008; 38(2): 223-228. http://dx.doi.org/10.1590/S004459672008000200005.

Chaves E, Fonseca W. Teca - Tectona grandis L. f. espécie de arbol de uso multiple en America Central. Turrialba: CATIE; 1991. 47 p. Série Técnica n. 179.

Cordero LDF, Kanninen M. Hacia el manejo intensivo de la teca (Tectona grandis) em Centroamérica. 2003 [citado em 2009 jul 1]. Disponível em: http://www.ots.ac.cr/ rdmcnfs/datasets/biblioteca/pdfs_private/nbina-780.pdf

Costa KL. Crescimento de Tectona grandis (Teca), em sistema silvicultural de talhadia composta em Minas Gerais [dissertação]. Lavras: Universidade Federal de Lavras; 2011. 107 p.

Drescher R. Crescimento e produção de Tectona grandis Linn F., em povoamentos jovens de duas regiões do estado de Mato Grosso - Brasil [tese]. Santa Maria: Universidade Federal de Santa Maria; 2004. 116 p.

Gonzaléz WF. Manual para productores de teca (Tectona grandis L. f) en Costa Rica. Heredia: FONAFIFO; 2004. $121 \mathrm{p}$.

Jerez M, Vicent L, Moret Y, Gonzáles R. Regímenes de espaciamiento inicial y aclareo en plantaciones de Teca
(Tectona grandis L.f.) en Venezuela. 2003 [citado em 2009 jul 2]. Disponível em: www.una.ac.cr/inis/docs/teca/ temas/RegmenesdEspaciamiento1.pdf

Kanninen M, Perez Cordero LD, Montero M, Viquez E. Intensity and timing of the first thinning of Tectona grandis plantations in Costa Rica: results of a thinning trial. Forest Ecology and Management 2004; 203(1): 89-99.

Macedo RLG, Gomes JE, Venturin N, Salgado BG. Desenvolvimento inicial de Tectona grandis L. f. (teca) em diferentes espaçamentos no município de Paracatu, MG. Revista Cerne 2005; 11(1): 61-69.

Oliveira CHR, Reis GG, Reis MGF, Xavier A, Stocks JJ. Área foliar e biomassa de plantas intactas e de brotações de plantas jovens de clone de eucalipto em sistemas agrossilvipastoris. Revista Árvore 2008; 32(1): 59-68.

Passos CAM, Bufulin L Jr, Gonçalves MR. Avaliação silvicultural de Tectona grandis L.f., em Cáceres - MT, Brasil: resultados preliminares. Ciência Florestal 2006; 16(2): 225-232.

Perez LD, Kanninen M. Stand growth scenarios for Tectona grandis plantations in Costa Rica. Forest Ecology and Management 2005; 210(1/3): 425-441.

Reis GG, Reis MGF. Fisiologia da brotação de eucalipto com ênfase nas suas relações hídricas. Série Técnica IPEF 1997; 11(30): 9-22.

Salas EC, González WF. Ensayos de aclareo y crecimiento en plantaciones de teca (Tectona grandis, L.f.) en la Península de Nicoya, Costa Rica. 2003 [citado em 2009 jul 2]. Disponível em www.una.ac.cr/inis/docs/teca/temas/ RAenTECA2.pdf

Schneider PR, Finger CAG, Hoppe JM, Drescher R, Scheeren LW, Mainardi G, Fleig FD. Produção de Eucalyptus grandis Hill ex Maiden em diferentes intensidades de desbaste. Ciência Florestal 1998; 8(1): 129-140. 